POROUS ORGANIC NETWORKS

Solid-state synthesis

Nat. Commun. 8, 1599 (2017)

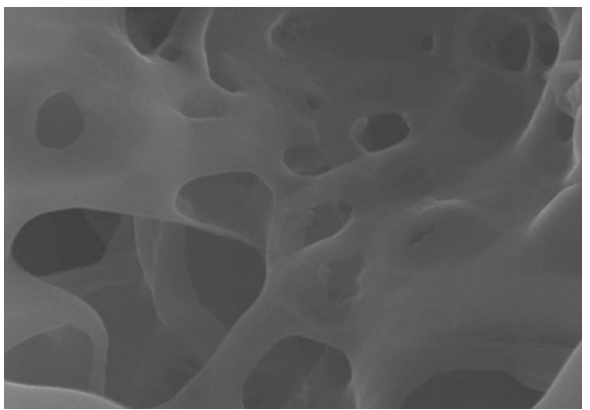

Credit: Macmillan Publishers Ltd

Researchers have synthesized various porous organic networks (PONs) with high surface area in liquid phase and demonstrated their capability in heterogeneous catalysis, gas capture and storage, energy storage and optoelectronics. However, no PONs have been obtained via solid-state reactions. Now, Jong-Beom Baek and colleagues from Ulsan National Institute of Science and Technology report the formation of a $3 \mathrm{D}$ PON via solidstate explosion of organic single crystals.

The key building block of the PON, 2,3,6, 7,14,15-hexaethynyl-9,10-dihydro-9,10-[1,2] benzenoanthracene (HEA), contains three enediyne groups, enabling it to self-polymerize via the Bergman reaction (cycloaromatization) to produce a $3 \mathrm{D}$ polyHEA. However, the occurrence of this reaction normally requires the presence of hydrogen donors in liquid phase, along with dicobalt octacarbonyl catalysts. Now, Baek et al. demonstrate that the HEA single crystals, consisting of HEA, two acetone molecules and one water molecule, can form a 3D PON in $0.11 \mathrm{~s}$ via an explosive solid-state reaction when the bulk crystals are heated with a heating gun. Recognizing the dominating effect of the primer molecules (acetone and water), they propose that these molecules can accumulate kinetic energy when heat is applied. Upon their sudden evacuation, the crystal lattices are disturbed and the enediyne groups become closer together, resulting in the formation of polyHEA via cycloaromatization. The synthesized polyHEA shows fish-net-type morphology and high thermal stability in air. Moreover, it exhibits distinguished $\mathrm{CO}_{2}$ adsorption capability due to the abundant electron-rich cavities.

https://doi.org/10.1038/s41565-017-0056-0

\section{CANCER NANOTECHNOLOGY \\ T-cell magnetic drive \\ ACS Nano. 11, 10724-10732 (2017)}

\section{Adoptive T-cell transfer is a cancer} immunotherapy strategy that involves extraction of T cells from a patient, ex vivo expansion and activation, and reinfusion in the body for tumour targeting. During the ex vivo treatment, T cells are trained to recognize cancer biomarkers on exposure to antigen-presenting cells (APC), which reprogrammes them into antigen-specific cytotoxic T cells (CTLs) for tumour recognition and killing. The use of natural APCs, however, hampers precise control over T-cell stimulation and often leads to low reproducibility and poor survival of the CTLs.

Now, Zhang et al. engineer an artificial APC that consists of an iron oxide magnetic nanocluster coated with a leukocyte membrane containing azide-modified lipids, which provide useful click-chemistry handles for functionalization with specific T-cell stimulation moieties. The leukocyte membrane also confers biocompatibility and endows the particles with a

\title{
2D MATERIALS
}

\section{Growing randomness}

A physically unclonable function (PUF) of a semiconducting device acts as its digital fingerprint, created as a result of manufacturing randomness. Silicon-based PUFs provide a CMOS-compatible solution, but their fabrication requires extensive postprocessing associated with increased security vulnerability of computing systems. Nanomaterials that exhibit randomness of nucleation during large-scale growth may prove to be promising cost-effective security primitives. A. Alharbi et al. now report unclonable cryptographic primitives obtained via standard chemical vapour deposition of a few-layer transition metal dichalcogenide (TMD), $\mathrm{MoS}_{2}$.

During the growth, multiple layered islands are simultaneously formed on a continuous $\mathrm{MoS}_{2}$ monolayer. Using statistical analysis of the surface coverage, the authors confirm the natural randomness of the speckle distribution. Next, a small region of the film is used to fabricate a physical security primitive consisting of $32 \times 64$ pixel dense arrays. Owing to the arbitrary thickness distribution of the film features and the thickness-sensitive excitonic emission of $\mathrm{MoS}_{2}$, on photoexcitation the primitive exhibits a unique optical response of the array into a binary cryptographic key. Such unclonable TMD-based primitives can be readily scaled to large-area manufacturing.

fluid coat that allows better interaction with the T cells. In comparison with activation using soluble antibodies, the authors show that their artificial APCs lead to more efficient T-cell expansion and to increased cytotoxicity. Leveraging on the magnetic properties of the APC-CTL complex, which is stable enough to survive intravenous injection in animals, the authors also show that they can direct the APC-CTL specifically to tumours and image its distribution within tissues using MRI. Delivery of the magnetically guided APC-CTL complex results in reduced tumour growth, minor side effects and increased animal survival when compared to controls receiving only CTLs or the APC-CTL complex with no magnetic guidance.

https://doi.org/10.1038/s41565-017-0055-1

\section{WATER TREATMENT}

No waste and no salt

Energy Environ. Sci. http://doi.org/chdn (2018)

Energy and water represent two pillars for human life. Societies endeavour to obtain new energy sources and clean, fresh water. Rather than pursue each separately, scientists have recently tied together wastewater treatment and desalination, harvesting energy from waste to remove salt from seawater. However, a common wastewater treatment strategy, using the microbial fuel cell, employs $\mathrm{pH}$ - and salt-sensitive organisms.

Now, Kim et al. report an abiotic waterenergy nexus that executes light-driven wastewater remediation, fuel production and seawater desalination. The researchers fabricated photoanodes, composed of hydrogentreated titanium dioxide nanorods, to absorb UV-light and undergo photochemistry. This treatment improved the photoelectrochemical performance by increasing the material conductivity and the nanorods' substrateoxidation abilities. The nanorod electrodes could oxidize chloride, producing urea-decomposing radicals, and simultaneously produce hydrogen gas $\left(\mathrm{H}_{2}\right)$ from the excess protons and electrons.

To test the solar water-energy nexus, the researchers built a three-chamber device, separating the anode and cathode compartments with a seawater-containing ion chamber between ion-selective membranes. The chloride diffused from the seawater to the anode and was consumed by nanorod photochemistry, while the sodium ions diffused into the cathode chamber to boost electrolyte conductivity and $\mathrm{H}_{2}$ evolution activity. Altogether, the photoelectrodes could completely remove urea within 12 hours, and $50 \%$ of seawater's salt at an $80 \%$ Faradaic yield for $\mathrm{H}_{2}$ evolution. $A W$ https://doi.org/10.1038/s41565-017-0057-z

Olga Bubnova, Chiara Pastore, Wenjie Sun, Adam Weingarten 